\title{
Prospettive e modelli per la qualità nell'insegnamento della lingua italiana come L2 e LS. Intervista a P.E. Balboni.
}

\author{
di Giuseppe Maugeri
}

\begin{abstract}
Nell'intervista che qui di seguito vi proponiamo, Paolo Balboni, docente di Didattica delle lingue moderne e direttore del Laboratorio ITALS dell'Universita' Ca' Foscari di Venezia, fa il punto sulle diverse strategie finalizzate a rendere più accessibile e spendibile la lingua e la cultura italiana all'estero, proponendo un modello di formazione che mira a valorizzare le competenze didattiche e sociali acquisite dai docenti grazie ai percorsi di aggiornamento glottodidattico del Laboratorio ITALS.

Keywords: Paolo Balboni. Glottodidattica. Lingua e cultura italiana. Laboratorio ITALS.
\end{abstract}

\section{RESUMO}

$\mathrm{Na}$ entrevista que apresentamos a seguir, Paolo Balboni, professor de Didática de línguas modernas e diretor do Laboratório ITALS da Universidade Ca' Foscari de Veneza, focaliza as diversas estratégias utilizadas no sentido de tornar mais acessíveis e utilizáveis a língua e a cultura italianas fora da Itália, propondo um modelo de formação que objetiva a valorização das competências didáticas e sociais adquiridas pelos professores graças aos percursos de atualização glotodidática do Laboratório ITALS.

Palavras-chave: Paolo Balboni. Glotodidática. Língua e cultura italiana. Laboratório ITALS.

\section{L'INTERVISTA}

Nell'odierna società delle conoscenze, l'educazione linguistica ha assunto un ruolo sempre più strategico, configurandosi man mano come area di mercato i cui processi formativi si adoperano per promuovere l'accesso ai cittadini a quelle abilità e a quei saperi integranti della vita 


\section{moderna. Secondo quali linee programmatiche e azioni la Comunità}

Europea affronta e realizza gli obiettivi di politica linguistica?

Anzitutto chiariamo il concetto di educazione linguistica: non è solo l'insegnamento delle lingue straniere (ad esempio il portoghese a un italiano), ma anche quello delle lingue materne (l'italiano a un madrelingua italiano) e seconde (l'italiano a immigrati). E' un grande mercato, probabilmente superiore a quello dell'alfabetizzazione e del continuo aggiornamento nella competenza informatica; ma è anche la chiave di una società aperta (gli stati europei sono aperti l'uno verso l'altro, ma l'apertura necessaria è superiore a quella di un piccolo continente come l'Europa) e giusta, in cui tutti abbiano pari opportunità di partenza.

L'Unione Europea non può intervenire sulla politica educativa dei singoli Stati, ma può fare raccomandazioni e può giocare su incentivi e finanziamenti. Ma di fatto può farlo solo per quel che riguarda le lingue europee, insegnate come lingue straniere nelle scuole e come lingue seconde in tutti i progetti di scambio tipo 'Erasmus' - ma può farlo usando come pretesto il fatto che il Trattato di Maastricht, da cui nasce l'Unione, prevede che tutti i cittadini europei imparino due lingue straniere (l'inglese e un'altra) e che l'interazione tra europei richiede un forte investimento nell'apprendimento/insegnamento linguistico. Sulla lingua nazionale e il suo insegnamento a madrelingua e immigrati l'Ue non può intervenire né ufficialmente né di fatto, perché ciascuno dei 27 paesi ha politiche e tradizioni e obiettivi differenti. Eppure una società giusta nasce anzitutto da una padronanza piena della madrelingua, nelle sue varietà, nei suoi registri; e la condizione di base perché un immigrato possa integrarsi e divenire una risorsa e non un problema è la padronanza piena, non solo nella comunicazione di base, della lingua del paese che lo accoglie. 
Ritiene che gli impegni formulati dalla politica linguistica della UE possano assicurare al sistema educativo italiano dei modelli formativi in grado di ridefinire i problemi interni, anche sulla base dei valori europei?

Come ho detto, la politica linguistica dell'UE è indiretta, e spesso si svolge con la collaborazione con un organismo culturale che raccoglie tutti gli stati europei, il Consiglio d'Europa. E' il CdE che ha lanciato nel 1967 il Progetto Lingue Moderne, che ha portato alla stesura di 22 'Livelli soglia', è il CdE che ha predisposto il Common European Framework e anche il European Language Porfolio, dove sono stati definiti i livelli A1, A2, B1 ecc.

Si tratta di impostazioni che hanno cambiato i materiali didattici, i programmi scolastici, la prassi di insegnamento.

In che modo i recenti tagli alla cultura incidono sulla capacità dei soggetti istituzionali, quali Scuola e Università, di progettare e pianificare degli interventi formativi in linea con le necessità e le richieste di formazione continua e di aggiornamento metodologico degli insegnanti?

Una razionalizzazione della spesa era necessaria, poteva far bene a tutto il sistema formativo, dove ci sono corporazioni, gruppi strutturati che impediscono l'innovazione. Ma i tagli 'lineari', cioè statistici (ad esempio, 10\% in meno a tutti) non premia gli eccellenti e non punisce gli scadenti. Questo è stato l'errore culturale dell'impostazione di Tremonti - errore anche politico: se fare politica vuol dire fare scelte, il governo ha rinunciato a scegliere, quindi a 
fare politica. Un esempio: si vuole ridurre del 30\% il costo delle università, come è stato deciso? bene: si dica alle università che hanno 3 anni per tagliare del 30\%, lasciando che sia ogni università a decidere cosa e come tagliare. Se invece succede che si agisce sui pensionamenti obbligati e sul blocco delle assunzioni, come è stato fatto, si lascia che a decidere sia la casualità dell'anagrafe: quanti centri eccellenti sono stati distrutti da una norma che mette in pensione anticipata i vecchi docenti e non consente di sostituirli? I danni non sono stati fatti dalla politica seguita, ma dalla mancanza di una politica intesa come capacità di scelta.

Qual è, a Suo modo di vedere, il nesso strutturale che collega l'evoluzione tecnologica con la formazione e la figura del docente di lingua impegnato a ridefinire e internazionalizzare il suo linguaggio di insegnamento?

L'insegnante deve presentare la lingua e creare condizioni perché essa venga esercitata: presentare la lingua solo su carta quando ci sono DVD è assurdo; far simulare un dialogo solo in aula quando esiste skype, è assurdo.

Ci potrebbe parlare delle nuove frontiere della formazione glottodidattica in riferimento a contesti d'uso che superano i limiti geografici dell'aula e della metodologia didattica che viene applicata?

Il principale problema nella formazione dei docenti non è più, tranne in pochi casi di tradizionalismo endemico che dovrebbe sparire con il pensionamento 
dei docenti, quello relativo all'approccio comunicativo, ma quello di accesso alla tecnologia e di mantenimento al passo con le sue evoluzioni.

Ma non è possibile tenere aggiornati tutti gli insegnanti quando ogni giorno compaiono nuove diavolerie tecnologiche: ma è possibile far sapere ai docenti che cosa può fare la nuova tecnologia, e poi lasciare che siano gli studenti (che non hanno alcun problema) a realizzare in pratica, sotto la guida del docente, un uso glottodidattico delle nuove macchine e dei nuovi software. L'insegnante deve sapere (e basta pochissimo a farglielo sapere) che cosa si può fare per le lingue con uno smart phone - e gli studenti, guidati sul cosa, non avranno problemi sul come.

In che modo le nuove metodologie delle formazione hanno attratto investimenti significativi da parte di Istituti e Università?

Non c'è una politica generale ma una politica dei singoli istituti e delle singole università, che sono per legge autonome in questo senso. Ma con i tagli di bilancio degli ultimi 10 anni parlare di investimenti significativi è fuori luogo. Si sono fatte cose stupende, ma solo dove si è trovato uno sponsor, o un preside interessato, del personale tecnico entusiasta - ma nulla di sistematico.

E' inusuale che nel panorama accademico italiano vi sia una struttura come il Laboratorio ITALS di cui Lei è direttore, che attraverso il lavoro di formazione e di ricerca si autofinanzi: secondo quale logica è stato elaborato il progetto ITALS e qual è il ruolo del Laboratorio nella produzione della ricerca glottodidattica? 
Itals è un'istituzione pubblica, quindi non ha fini di guadagno: tutto quello che rimane dai vari corsi descritti in www.itals.it viene dedicato al finanziamento della ricerca... e dei ricercatori!

Sulla ricerca Itals, basta vedere la bibliografia nel sito indicato sopra. Sono centinaia di saggi e volumi.

Ma oltre a quella, c'è anche la rivista Itals. Didattica e linguistica dell'italiano a stranieri, e ci sono altre riviste dove la presenza del corpus di ricercatori cresciuti in Itals è molto presente, da Scuola e lingue moderne a In.It, e poi ci sono le collane presso Utet, Guerra, Cafoscarina, Aracne.

A parte la quantità di ricerca, è la sua natura ad essere interessante: Itals ha una visione complessa della glottodidattica, intesa non solo come linguistica applicata o educativa, ma come scienza complessa che include contributi delle scienze neuro-psicologiche, di quelle socio-antropologiche, di quelle pedagogiche. Una visione complessa che vuole dare una risposta alla necessità di una glottodidattica adeguata alla società complessa in cui viviamo.

\section{In quale direzione l'ITALS e la sua offerta si stanno sviluppando?}

Due direzioni: una ricerca sempre più spinta ad esplorare i 'margini' della glottodidattiche, quelle aree borderline dove si individuano nuovi territori da esplorare - dalla glottodidattica delle lingue a dislessici, all'insegnamento delle lingue attraverso gli smartphone, dall'uso di canzoni e sport per insegnare la lingua, al ruolo che può avere un contributo spesso trascurato dell'Italia al mondo, l'opera lirica.

Sempre con attenzione all'equilibrio tra le due componenti: quella teorica, che vuole studiare e descrivere la realtà, e quella operativa, che deve tradurre le ricerche anche in strumenti d'azione didattica. 


\section{La qualità è il criterio di differenziazione delle offerte ITALS: in che cosa consiste il progetto ITALS per la qualità didattica?}

La filosofia della qualità è quella del miglioramento continuo verso l'eccellenza. In particolar modo, il progetto ITALS per la qualità didattica si rivolge a Istituzioni e docenti che operano nell'ambito dell'insegnamento dell'italiano a studenti stranieri all'estero o in Italia.

Si tratta, in sintesi, di una sorta di marchio di qualità che attesta la conformità delle strutture, delle risorse umane, dei processi alle indicazioni proposte per creare un vero e proprio passaporto professionale e di accreditamento didattico. Chi chiede di accreditarsi, inizia sotto la guida di ITALS un percorso di miglioramento continuo basato, ad esempio, su percorsi di formazione per i docenti, di gestione dell'offerta didattica di un'istituzione, ecc.

Nel sito http://venus.unive.it/progetto_qualita vengono raccolti i dati relativi agli insegnanti e alle organizzazioni che si sottopongono al processo di valutazione per l'accreditamento.

Nel sito si trovano anche i criteri relativi al processo di accreditamento, i moduli per la richiesta e molte altre informazioni.

\section{In base a quali requisiti e caratteristiche un corsista può far parte e rafforzare il senso di appartenenza alla comunità ITALS? \\ Il requisito è semplice: fantasia (tanta!), disponibilità ad esplorare quelle che apparentemente sono follie, desiderio di mettersi in gioco; ma insieme a queste caratteristiche personali, serve la convinzione che la fantasia senza uno studio teorico solido è solo un giochino sterile che non porta da nessuna parte.}




\section{Fonti bibliografiche e sitografiche}

P.E. Balboni, Le sfide di Babele. Insegnare le lingue nelle societa' complesse, UTET Universita', Torino 2008.

P.E. Balboni, Fare educazione linguistica. Attivita' didattiche per italiano L1 e L2, lingue straniere e lingue classiche, UTET Universita', 2008.

P.E. Balboni, R. Dolci, G. Serragiotto, ITALS: dieci anni di formazione e ricerca, Bonacci editore, Roma, 2007.

P.E. Balboni, Dizionario di glottodidattica, Guerra Edizioni, Perugia, 1999.

F. Caon, Educazione linguistica e differenziazione. Gestire eccellenza e difficolta', UTET Universita', Torino, 2008.

M. Mezzadri, Integrazione linguistica in Europa, UTET Universita', Torino, 2006.

G. Serragiotto, Sillabo di riferimento per la formazione degli insegnanti di italiano a stranieri, Libreria Editrice Cafoscarina, Venezia, 2009. 